\title{
Advancing Downward to the Basic Level
}

\author{
Jean M. Mandler \\ Department of Cognitive Science \\ University of California, San Diego \\ Laraine McDonough \\ Department of Psychology
}

Brooklyn College and The City University of New York Graduate Center

\begin{abstract}
In 3 experiments, we studied whether infants and young children understand various basic-level conceptual distinctions in the domains of household artifacts, animals, and vehicles. Using small replicas, we modeled events such as washing dishes in a sink for children 14, 19, and 24 months old, and then gave them an exemplar from the same basic-level concept (another sink) and an exemplar of another concept from the same domain (bathtub). We measured which object they used to imitate the event. Fourteen-month-olds did not differentiate among basic-level categories in any of these domains, for example, washing dishes in both a tub and a sink, and putting both a rabbit and a bird in a nest. By 19 months, inappropriate behavior was greatly reduced for household artifacts and for vehicles, but not for animals. By 24 months, performance was mainly appropriate for all 3 domains. It was also shown that although 14-month-olds are not making many conceptual distinctions at the basic level, they are nevertheless beginning to make some broader conceptual distinctions among artifacts.
\end{abstract}

A common view of concept development in the psychological literature is that the first concepts to be formed are at what is usually termed the basic level (Mervis \& Rosch, 1981; Rosch, Mervis, Gray, Johnson, \& Boyes-Braem, 1976). This level of conceptualization has never been unambiguously defined (Lassaline, Wisniewski, \& Medin, 1992; Mandler, 1997; Tanaka \& Taylor, 1991) but is usually understood by example. Dog, table, car, and cup are said to be basic-level concepts. Animal (or

Requests for reprints should be sent to Jean M. Mandler, Department of Cognitive Science, University of California, San Diego, La Jolla, California 92093-0515. E-mail: jmandler@ucsd.edu 
mammal), furniture, vehicle, and utensil are said to be superordinate concepts. Until recently, the hypothesis that basic-level concepts are the first to be formed was typically tested with children 3 years old or older (e.g., Rosch et al., 1976). In the past decade, however, research has begun to show that concept formation has its origins in infancy, and in our laboratory we have conducted a number of experiments indicating that the earliest concepts are broader than the level of dog, table, car, and cup; instead they appear to be at the level of animal (or mammal), furniture, vehicle, and utensil (Mandler \& McDonough, 1993, 1996, 1998a, 1998b).

In our most recent series of experiments investigating concept formation in infancy, we used a generalized imitation technique (Mandler \& McDonough, 1996, 1998b; McDonough \& Mandler, 1998; see also Bauer \& Dow, 1994). We model a simple event using small replicas of real-world objects and encourage infants to imitate what they have observed. For example, we might show a dog being given a drink from a cup. Then we give the infants the cup, but instead of the dog used for modeling we give them two other objects instead. Along with the cup, we might give them a rabbit and a car or a different dog and a cat, to see which object they choose for their imitations. By varying the selections available to the infants, we are able to test what they have understood from the event they have observed.

The generalized imitation technique as a method of assessing infant knowledge relies on three characteristics of infant imitation. First, infants imitate spontaneously and do not need instructions to do so. Second, infants treat realistic little models as representations of real-world objects and understand familiar activities modeled with objects as representing real-world events (Mandler \& McDonough, 1998b; McDonough \& Mandler, 1998). At first glance, this may seem surprising in 1-year-old infants, but it reflects the same capacity that is manifested in recognizing pictures and that a few months later is expressed in symbolic play. ${ }^{1}$ One illustration of the representational nature of infants' imitations is the fact that they tend not to imitate events they think are incorrect (Bauer \& Thal, 1990; Killen \& Uzgiris, 1981). For example, Bauer and Thal (1990) found that 21-month-olds were less likely to imitate when event sequences were modeled in the incorrect order and when they did imitate they often reproduced the sequences in the correct order instead. Mandler and McDonough (1996) found that 14-month-olds rarely would imitate incorrect actions such as giving a car a drink or keying a dog; they would imitate the experimenter doing an appropriate action on an unfamiliar exemplar of a class (e.g., giving an aardvark a drink), but would not do something inappropriate to the class.

\footnotetext{
${ }^{1}$ Imitation of events occurs earlier in ontogeny than symbolic play, presumably because the infant does not need to retrieve an event from long-term memory. The model provides the infant with the information about what is to be done.
} 
Third, because infants reproduce what they have conceptually understood from watching a model, when this understanding is limited, their imitations are often only approximate, as shown by the extensive observations of Piaget (1951). The generalized imitation task takes advantage of this fact by letting the infants choose which objects to use to imitate. The available choices can be more or less related to the modeled act, and so the objects the infants choose provide information about what they have understood. For example, when we modeled a dog drinking from a cup and then gave infants the cup along with another animal and a vehicle, they almost always used the other animal for their imitations (Mandler \& McDonough, 1996). This finding indicates that they understood that animals drink but vehicles do not. Furthermore, if we gave them another dog and a cat, they were as likely to use the cat as the dog to imitate what they had seen (Mandler \& McDonough, 1998b). This finding also indicates that infants understand that animals drink, but in addition that they may have construed the event they watched not so much as a dog drinking but in a more general way as an animal drinking. On the other hand, if we gave them another dog and a bird along with the cup, they were more likely to use the other dog (Mandler \& McDonough, 1998b). This additional finding suggests that the infants construed the event they watched not in the most general fashion as an animal drinking nor in a basic-level fashion as a dog drinking, but at an intermediate level of generality, perhaps as a land animal or a legged animal drinking. Thus, this technique allows us to assess not only how far infants generalize behavior but also how they have construed the events that they have observed.

Using this technique, we have found that infants between 9 and 14 months old tend to generalize observed behavior very broadly. In many instances they generalize observed behavior to the limits of the domain to which the modeled object belongs. Thus, when we model a behavior such as drinking or sleeping with an animal, they generalize it to all animals, including fish and aardvarks (Mandler \& McDonough, 1996). When we model an action such as keying a car, they generalize it to all vehicles, including forklifts and airplanes. These generalizations, which go beyond any observations they have made in everyday life, suggest that infants in this age range have some general (perhaps rather vague) conceptions of what animals and vehicles are, and make their generalizations about the properties of these domains on the basis of these broad conceptions. For example, it has been hypothesized that infants' first conception of animals is as self-movers that interact contingently with other objects (e.g., Legerstee, 1992; Mandler, 1992; Molina, Spelke, \& King, 1996; Poulin-Dubois, Lepage, \& Ferland, 1996). When they observe people, and perhaps a dog or cat drink, they make the generalization that all self-moving, contingent interactors drink. Thus, they are not dependent on the details of what a particular object looks like to form an initial concept of animal (or animate thing) but rather on observing certain broad characteristics that animate objects exhibit in their movements and interactions with other objects (Nelson, 1974). 
This approach enables infants to begin building a conceptual system, but obviously they are going to have to learn much more precise information about objects than their superordinate category membership. Infants will need to learn how one animal behaves differently from another (domestic cats are generally safe but dogs are iffy) and the many cultural differences in the use of artifacts (we drink from cups and glasses, not from cooking pots, even though cooking pots are good containers). This means that at some point they are going to have to pay attention to the relation between the finer details of what animals and kitchen utensils look like and their typical behaviors or functions. We know that infants can see the differences between one kind of animal and another (Eimas \& Quinn, 1994) and between one kind of furniture and another (Behl-Chadha, 1996). However, that does not mean that they have learned what these things are or what they do. Even 3-month-olds categorize dogs as different from cats (Quinn, Eimas, \& Rosenkrantz, 1993), but they do not seem to draw any conceptual implications from those differences until some months later (Mandler \& McDonough, 1998b). Similarly, 3-month-olds categorize tables as different from chairs but do not thereby necessarily understand their different functions. For this reason it is important to test exactly what infants do know at different stages of development about the differences between dogs and rabbits or between bathtubs and beds other than that they look different from each other.

In most of the studies we conducted on early concept formation, we investigated knowledge about behavior that is characteristic of whole domains, such as that animals eat or vehicles give rides. These data demonstrated that infants are making broad generalizations about these domains, but does not preclude the possibility that they are learning properties associated with specific basic-level classes as well. For example, when do infants learn the behavior specific to particular animals, such as that dogs but not birds chew on bones, or the specialized uses of particular kinds of furniture, such as that beds but not bathtubs are used for sleeping? Our hypothesis about learning basic-level properties such as these was that they too would at first be overgeneralized in the same way that more general properties such as drinking and sleeping were generalized to fish and keying to airplanes (Mandler \& McDonough, 1996). Another way of stating this hypothesis is to say that because at first infants construe objects such as dogs as animals, not as dogs, they will overgeneralize dog behavior to other animals, or because they construe objects such as cups as containers they will overgeneralize drinking from cups to drinking from other containers as well.

In our first examination of this hypothesis we examined two natural kind and two artifact properties associated with basic-level kinds (Mandler \& McDonough, 1998b). For the animal properties, we modeled a dog chewing on a bone and tested generalization with another dog and a goose. We also modeled sniffing a flower and tested generalization with another flower and a tree. For the artifact properties we modeled drinking with a cup and tested generalization with a mug and a frying 
pan. We also modeled sleeping in a crib and tested generalization with a bed and a bathtub. At 14 months, the infants generalized these basic-level properties to both appropriate and inappropriate exemplars, and this happened for both the natural kinds and artifacts. At 19 months, they still generalized the natural kind properties equally often to appropriate and inappropriate objects, but had begun to narrow down their responses in the artifact domains. That is, they still made a goose chew on bones and a person sniff trees, but now they were more likely to restrict drinking to a cup (not a pan) and sleeping to a bed (not a bathtub).

The experiments reported here explored the growth of conceptual knowledge in the domains of household artifacts, vehicles, and animals in more detail. The experiments again used the generalized imitation technique to examine the differentiation of these global domains into smaller conceptual classes. Actions such as putting a bird in a nest were modeled. Infants were not given the same bird as used in the modeling but instead were given a choice of another bird or a rabbit to use for their imitations. It was hypothesized that younger infants would respond mainly at a global level, treating various subclasses of a domain as equivalent in terms of how the objects are treated or what they do, and that only older infants would respond appropriately at the basic level.

In our previous work, differentiation began earlier in the realm of household artifacts than for animals and plants (Mandler \& McDonough, 1998b), but we only tested two household items. Therefore, we decided first to assess more household basic-level properties to see if the developmental course we found was broadly replicable. The properties in question were all actions associated with specific artifacts (e.g., brushing hair with a hairbrush). The question asked was whether infants overgeneralize these actions to related artifacts in the same domain. Because in the previous work even 19-month-olds were not performing perfectly on the generalization tasks, in Experiment 1 we tested 14-, 19-, and 24-month-olds. Experiment 2 was a control experiment to show that 14-month-olds, who fail these basic-level tests of knowledge about the function of specific household artifacts, succeed on the same tests if they are given conceptually more differentiated contrasts.

In Experiment 3, we compared differentiation in the vehicle and animal domains. In some (but not all) prior work we found earlier differentiation in the vehicle domain than in the animal domain. We found that, even at 7 months, infants were making some distinctions among different kinds of vehicles, whereas they were treating all animals alike (Mandler \& McDonough 1993). We also found that although 14-month-olds were undiscriminating in their choice of animals to imitate an event, they were somewhat more likely to choose another vehicle at the same basic level for their imitations (Mandler \& McDonough, 1998b). Therefore, in Experiment 3 we studied further the question of whether infants are more advanced in the vehicle domain by testing their understanding of actions associated with specific vehicles. We tested 14-, 19-, and 24-month-olds on their knowledge of basic-level vehicle properties and contrasted these with their knowledge of basic-level animal properties. 


\section{EXPERIMENT 1}

\section{Method}

Participants. Twenty-four participants were tested, 8 in each of three age groups: 14-, 19-, and 24-month olds. The mean ages were 14 months, 12 days (range $=14$ months, 0 days -14 months, 23 days); 19 months, 14 days (range $=19$ months, 3 days -20 months, 0 days); and 24 months, 18 days (range $=24$ months, 6 days-25 months, 4 days). An equal number of boys and girls were tested at 14 and 19 months old. Five boys and 3 girls were tested at 24 months. A small toy was given to the children for their participation. Participants were recruited from an existing pool of volunteer parents who had responded to advertisements in local newspapers. Although records were not kept on the ethnic backgrounds of the specific participants in this and the following two experiments, the records of participation in our studies overall reflect a distribution similar to the San Diego area in general (75\% White, $10 \%$ Hispanic, $8 \%$ Asian, $6 \%$ Black, and 1\% other). Most participants come from middle-class homes.

Objects and properties tested. The participants were tested on four events, each of which demonstrated a property characteristic of a basic-level category in the household domain: washing a plate in a sink, sitting on a chair at a dining table, brushing hair with a hairbrush, and pounding with a hammer. All properties were tested using miniature replicas of the objects typically used for these activities. Vocalizations accompanied the experimenter's modeling of each property. Washing a dinner plate ("Wash the dishes!") was modeled using a kitchen sink that was set into a countertop. The sink was partitioned into two halves and had a swivel tap. The experimenter placed the plate into the sink and then pretended to wash it off with two fingers. Generalization was tested with a different kitchen sink (also with a divided sink and with a dishwasher under the counter) and a bathtub that had separate hot and cold taps. Sitting at a dining table (on which were painted two table settings consisting of plates and flatware) was demonstrated by putting a small doll on a ladder-back dining chair and placing it at the table, saying, "Time to eat!" Generalization was tested with a different chair and a toilet (with the lid glued in the up position). Brushing hair (“Ooooh, nice!") was modeled using a hairbrush and a puppet with a tuft of hair atop its head. Generalization was tested with another hairbrush and a toothbrush. Hammering ("Bam, bam, all fixed!") was demonstrated with one pipe that was placed atop another and pounded together with a hammer. Generalization was tested with a different hammer and a wrench.

Procedure. Participants were invited into a laboratory set up as a playroom. After a brief warm-up period, they were seated either in a child's seat or in the parent's lap across the table from the experimenter. Parents were asked not to assist 
their children in any way throughout the session. Two warm-up tasks, designed to accustom the participants to the imitation procedure, were administered prior to the generalization task. One was modeled by placing a plastic star into a container, covering the container, and shaking it; the other was modeled by putting a spatula in a frying pan and stirring. Participants were encouraged to imitate both actions and were praised for doing so.

Participants' demonstrations of the properties were evaluated twice-once before the properties were modeled (baseline) and again after they were modeled (generalization).

For the baseline measurement, before each property was modeled, participants were given both test exemplars (e.g., sink and bathtub) and the prop used to demonstrate the target action (the plate). The experimenter determined the amount of time for baseline exploration on the basis of whether the infant was still actively engaged with the objects. If an infant ignored any of the three objects, the experimenter would point it out saying "Look, did you see this one?" When the infant stopped responding to the objects, the experimenter took them away. The prop was left on the table and the test exemplars were placed out of sight. The experimenter then brought out the modeling exemplar (the other sink) and demonstrated the target action with the prop (the plate) three times with the appropriate vocalization ("Wash the dishes"). The experimenter then removed the modeling sink from the table so that it could no longer be seen.

In the generalization evaluation, the test exemplars (in this case the bathtub and the sink used at baseline) were brought back and simultaneously placed on the table to either side of the infant (with side placement of the appropriate and inappropriate exemplar counterbalanced across tasks). The experimenter then handed the prop (i.e., the plate) to the infant while repeating the vocalization ("Wash the dishes"). The experimenter then waited until the infant no longer responded to the objects before removing them. This procedure continued until each infant had been tested on all four properties. The order in which the properties were administered was counterbalanced across participants.

Scoring. Each infant was videotaped in this and the next two experiments. Participants were coded for performance (or nonperformance) of the modeled actions and whether the exemplar they used for doing so was appropriate or inappropriate. Washing dishes was scored if the infant put the plate in either the sink or bathtub but only scored as appropriate if the infant put the plate in the sink. Sitting at the table was scored if the infant put the doll either on the chair or the toilet but was scored as appropriate only if the doll was placed on the chair. Brushing hair was scored if the infant put either brush to the puppet's hair, but was scored as appropriate only if the hairbrush was used. Finally, hammering was scored if the infant banged the pipes with either tool, but was scored as appropriate only if the infant used the hammer rather than the wrench. Two coders were trained on data from our 
TABLE 1

Mean Number and Percentage of First Choices of Appropriate and Inappropriate Objects in Baseline and Generalization

\begin{tabular}{|c|c|c|c|c|c|c|c|c|c|c|c|c|}
\hline \multirow[b]{3}{*}{ Age in Months } & \multicolumn{6}{|c|}{ Baseline } & \multicolumn{6}{|c|}{ Generalization } \\
\hline & \multicolumn{3}{|c|}{ Appropriate } & \multicolumn{3}{|c|}{ Inappropriate } & \multicolumn{3}{|c|}{ Appropriate } & \multicolumn{3}{|c|}{ Inappropriate } \\
\hline & No. & $S E$ & $\%$ & No. & $S E$ & $\%$ & No. & $S E$ & $\%$ & No. & $S E$ & $\%$ \\
\hline \multicolumn{13}{|c|}{$\begin{array}{l}\text { Experiment 1: } \\
\quad \text { Household artifacts }\end{array}$} \\
\hline 14 & 1.00 & .33 & 25 & 0.88 & .35 & 22 & 1.25 & .31 & 28 & 1.63 & .32 & 41 \\
\hline 19 & 1.25 & .31 & 31 & 1.25 & .41 & 31 & 2.75 & .41 & 69 & 1.00 & .38 & 25 \\
\hline 24 & 1.75 & .25 & 44 & 1.25 & .31 & 31 & 3.00 & .33 & 75 & 1.00 & .33 & 25 \\
\hline \multicolumn{13}{|c|}{$\begin{array}{l}\text { Experiment 2: } \\
\quad \text { Household } \text { artifacts }^{\mathrm{a}}\end{array}$} \\
\hline 14 & 1.88 & .40 & 47 & 1.13 & .35 & 28 & 2.63 & .18 & 66 & 0.50 & .50 & 12 \\
\hline \multicolumn{13}{|c|}{$\begin{array}{l}\text { Experiment 3: } \\
\text { Animals and vehicles }{ }^{\mathrm{b}} \\
\text { Animals }\end{array}$} \\
\hline 14 & 0.38 & .18 & 19 & 0.50 & .19 & 25 & 1.00 & .27 & 50 & 0.25 & .16 & 13 \\
\hline 19 & 0.75 & .25 & 38 & 0.50 & .19 & 25 & 1.00 & .19 & 50 & 1.00 & .19 & 50 \\
\hline 24 & 0.50 & .27 & 25 & 1.00 & .33 & 50 & 1.63 & .18 & 82 & 0.13 & .13 & 7 \\
\hline \multicolumn{13}{|l|}{ Vehicles } \\
\hline 14 & 0.00 & .00 & 0 & 0.13 & .13 & 7 & 0.63 & .18 & 32 & 0.13 & .13 & 7 \\
\hline 19 & 0.25 & .16 & 13 & 0.00 & .00 & 0 & 1.63 & .18 & 82 & 0.25 & .16 & 13 \\
\hline 24 & 0.25 & .16 & 13 & 0.00 & .00 & 0 & 1.75 & .16 & 88 & 0.13 & .13 & 7 \\
\hline
\end{tabular}

${ }^{\mathrm{a}}$ maximum score $=4 .{ }^{\mathrm{b}}$ maximum score $=2$.

previous experiments until at least $95 \%$ agreement with previous coders was obtained. Then the coders coded every participant. The actions turned out to be unambiguous: Agreement between the two coders was $98 \%$. The few disagreements were resolved by a third coder. Coders were unaware of the hypotheses of the experiment, but not the acts that were modeled (they were obvious given the props being used and the children's own actions during the test).

\section{Results}

The top portion of Table 1 shows for each age group the mean number of times the appropriate or inappropriate object was chosen first during baseline and after the target action was modeled (i.e., at generalization) along with the standard error for each mean. The scores could range from 0 to 4 . The means are also expressed as percentages to facilitate comparisons across the three experiments. The percentages of appropriate and inappropriate responses in each period do not add to $100 \%$ 
because no participant performed a target action for every event during baseline and, except for the 24-month-olds, participants did not always perform a target action for every event during generalization either.

Despite not always performing the target action, appropriate and inappropriate choices were not entirely independent because every appropriate first choice limited the possible number of inappropriate ones (and vice versa). Therefore, the main analyses used difference scores, which were calculated by subtracting the number of inappropriate first choices from the number of appropriate first choices for each participant (range $=-4$ to +4 ). The means of these difference scores, which were computed separately for baseline and for generalization, along with their standard errors, are shown for each age group in the top portion of Table 2. The generalization data are reported first because the primary interest in this experiment is the extent to which children generalized from the modeled behavior to appropriate versus inappropriate objects.

Generalization analyses. The difference scores for choices in generalization (see Table 2) were entered into an analysis of variance (ANOVA) with age (14-, 19-, 24-month-olds) and gender as between-subjects variables. Follow-up analyses using $t$ tests were two-tailed in this and the following experiments. A main effect for age was found, $F(2,18)=5.77, p<.02$, indicating that the difference be-

TABLE 2

Mean Difference Between First Choice of Appropriate and Inappropriate Objects in Baseline and Generalization

\begin{tabular}{|c|c|c|c|c|}
\hline \multirow[b]{2}{*}{ Age in Months } & \multicolumn{2}{|c|}{ Baseline } & \multicolumn{2}{|c|}{ Generalization } \\
\hline & $M$ & $S E$ & $M$ & $S E$ \\
\hline \multicolumn{5}{|c|}{ Experiment 1: Household artifacts ${ }^{\mathrm{a}}$} \\
\hline 14 & 0.13 & .55 & -0.38 & .60 \\
\hline 19 & 0.00 & .60 & 1.75 & .77 \\
\hline 24 & 0.50 & .46 & 2.00 & .65 \\
\hline \multicolumn{5}{|c|}{ Experiment 2: Household artifacts $\mathrm{a}$} \\
\hline 14 & 0.75 & .59 & 2.13 & .30 \\
\hline \multicolumn{5}{|c|}{ Experiment 3: Animals and vehicles ${ }^{b}$} \\
\hline \multicolumn{5}{|l|}{ Animals } \\
\hline 14 & -0.13 & .30 & 0.75 & .37 \\
\hline 19 & 0.25 & .41 & 0.00 & .38 \\
\hline 24 & -0.50 & .53 & 1.50 & .27 \\
\hline \multicolumn{5}{|l|}{ Vehicles } \\
\hline 14 & -0.13 & .13 & 0.50 & .27 \\
\hline 19 & 0.26 & .16 & 1.38 & .32 \\
\hline 24 & 0.25 & .16 & 1.63 & .26 \\
\hline
\end{tabular}

${ }^{\mathrm{a}}$ maximum score $=4 .{ }^{\mathrm{b}}$ maximum score $=2$. 
tween appropriate and inappropriate generalization increased with age. Pairwise comparisons showed that the difference scores did not significantly differ between the 19- and 24-month olds, $t(14)=0.25, p=.81$; however, both were significantly greater than those of the 14-month-olds, $p \mathrm{~s}<.05$. There was also a main effect for gender, $F(1,18)=6.76, p<.02$. Gender did not interact with age: Girls $(M=2.00)$ performed more correctly overall than boys $(M=0.38)$ at all three ages.

If the children had no preference for the appropriate over the inappropriate exemplar, the difference scores should not be significantly greater than zero. To test this, we calculated one-sample $t$ tests against the chance score of zero for each gender. For girls, the difference scores were significantly greater than zero, $t(10)=$ $3.09, p<.02$, but for boys they were not, $t(12)=0.73$. The boys performed as many target actions as girls, so they were engaged in the task, but they were more indiscriminant in the objects they chose to carry out the activities.

We also calculated $t$ tests against zero for each age group. The 14-month-olds made more generalizations to the inappropriate than appropriate exemplars giving them a negative score $(M=-0.38)$, although this did not significantly differ from zero, $t(7)=-0.84, p=.43$. Thus, the 14 -month-olds in general were indiscriminant in the object they chose to imitate the modeled event. The mean difference score for the 19 -month-olds $(M=1.75)$ was marginally significant, $t(7)=2.26, p=.06$, and the mean difference score for the 24-month-olds $(M=2.0)$ was statistically significant, $t(7)=3.05, p<.05$. These results indicate that, on the whole, the basic-level properties were generalized appropriately by the two older age groups. Because difference scores are derivative measures, it might be thought that the increase in the size of the difference scores with age could be due not to more accurate target behavior but to an increasing willingness to imitate in general. However, inspection of Table 1 makes clear that the increase in responding that occurred with age was due to increased appropriate choices only; there was no increase in inappropriate choices. Therefore, the difference scores present a reasonable picture of the more accurate responding shown by the older children.

The basic finding that younger infants often used an inappropriate object to carry out target actions was pervasive and not due to poor performance by just a few of the participants. The 14-month-olds had a mean of 2.87 imitations (out of 4 ) across the four activities, but three quarters of the participants performed only one or none of these target actions with the correct objects. The 19-month-olds had a mean of 3.75 imitations, but by this age only one quarter of them performed only one action correctly (there were no zero scores). The rest performed three or more correctly. All 24-month-olds imitated on all four tasks and none of them performed as few as one action correctly. Thus, there was a straightforward developmental trend from overgeneralization at 14 months toward basic-level performance at 24 months.

Baseline analyses. Although in baseline the children had no particular reason to engage in the activities that were later modeled, as can be seen in Table 1, at 
all three ages they did carry out a moderate number of the target actions during spontaneous play. The baseline difference scores, computed between first choice of an appropriate object for the action versus an inappropriate one (see Table 2), were analyzed by a $t$ test at each age. None of the difference scores at any age were significantly greater than zero. Thus, before modeling the children performed target behaviors on appropriate and inappropriate objects at roughly the same rate. In addition, the baseline difference scores were subjected to an ANOVA with gender and age as between-subject variables. No significant effects were found, indicating that approximately the same amount of spontaneous demonstrations of the actions occurred at all ages and for both boys and girls.

Comparison of baseline to generalization and individual task analyses. To examine the effects of modeling the target activity on the participants' behavior, we used the number of appropriate first choices (out of 4) shown in Table 1. An ANOVA was conducted, with condition (baseline, generalization) as a within-subjects factor, and age and gender as between-subjects factors. There was a main effect of condition, showing that significantly more appropriate objects were chosen at generalization $(M=2.36)$ than at baseline $(M$ $=1.31), F(1,18)=18.84, p<.001$. Although, as can be seen in Table 1 , the mean number of appropriate first choices following modeling increased considerably with age, neither age nor Age $\times$ Condition interaction was significant in the ANOVA. There was also no main effect of gender; however, gender interacted with condition, $F(1,18)=6.20, p<.03$. At baseline, there was no significant difference between girls $(M=1.09)$ and boys $(M=1.54), t(22)=0.63$, but girls $(M=$ 2.73) were more accurate than boys $(M=2.00)$ in generalization, $t(22)=1.96, p$ $=.06$.

Finally, the data were examined to see if performance was better on some of the four tasks than others. An ANOVA was conducted with target action (washing dish, sitting at table, brushing hair, hammering pipes) as a within-subjects factor and age as a between-subjects factor. The dependent measure was the number of times an action was first demonstrated correctly during baseline and generalization combined (range $=0-2$ ). No significant effects of target action were found, $F(3,19)=1.44, p=.26$, suggesting that knowledge (or lack thereof) of the various basic-level properties was roughly the same for all four tasks.

\section{Discussion}

These data expand our prior finding that 14-month-olds have not yet identified specific basic-level properties for many categories of household artifacts (Mandler \& McDonough, 1998b). By 19 months, the majority of children have learned the relevant associations sufficiently well to narrow their choice of object to the appropri- 
ate category. In our previous study, 14-month-olds did not differentiate appropriate drinking containers from inappropriate ones (frying pans) or appropriate pieces of furniture in which to sleep from inappropriate ones (bathtubs). In this experiment, we found that 14-month-olds overgeneralized their responses to other household items as well. They used both a toothbrush and hairbrush to brush hair, put a dish into both a sink and a bathtub, hammered with both a hammer and a wrench, and put a little person on a toilet as well as a chair at a table.

Our previous work (Mandler \& McDonough, 1998b) indicated that 14-month-olds construe household objects in rather general ways, considering any small container as being drinkable from and various large household containers as being places to sleep. The findings of this experiment suggest that infants at this age also construe large household containers as being places to immerse objects, flat-surfaced furniture as places to sit, any kind of tool as suitable for hammering, and any kind of brush suitable for making hair look nice. Of course, these descriptions are our adult glosses and may be incorrect in their particulars. We are using them to capture the overly general character of what infants have understood about these objects. Our data suggest that when in their play 1-year-olds drink from a cup or put a doll in a bed or bang a peg with a plastic hammer we may attribute too much knowledge to them to say that they understand that cups are for drinking, beds are for sleeping, or that a hammer has a specific function. Their understanding may be more global and less detailed than such descriptions suggest. It is not until 19 months that we found a narrowing down of general characterizations to the more detailed specifications implied by the notion of basic-level concepts.

The data indicated that boys were slower in this development than girls. Of course, we cannot rule out the possibility that the boys were merely less attentive to detail in the context of an imitation task than were the girls. However, this alternative hypothesis seems unlikely for two reasons. First, there is a good deal of evidence that neither male nor female infants pick objects at random to imitate what they observe. At 14 months both boys and girls rarely choose objects from a different domain to imitate modeled events and they typically refuse to imitate behavior they consider to be incorrect (Mandler \& McDonough, 1996; McDonough \& Mandler, 1998). Second, there were no gender differences in Experiment 2 or (with one minor exception) in Experiment 3 in which animals and vehicles were tested. Therefore, the lack of selectivity in the boys' performance in this experiment appears to be due to indifference to basic-level distinctions in the household domain, such as found between one kind of brush and another or one kind of household container and another. This indifference was general for the 14-month-olds but largely disappeared by 19 months for the girls.

There is also the possibility that 14-month-olds might understand some of these properties in a different way than the glosses of "being drinkable from" or "making hair look nice" suggest. They might be based on more neutral physical descriptions of the various activities. For example, any flat surface of a certain height affords sit- 
ting and any small elongated object can be run across the hair. Therefore, we conducted a second experiment with 14-month-old participants, in which the same four properties were modeled but the choices provided by the test objects were conceptually more differentiated ones. All objects were artifacts, but not from closely related categories. For example, when washing was modeled, the test objects consisted of a sink and a bed. If 14-month-olds have a general idea of what washing involves (e.g., a container associated with water), even if they are not yet sure of the different cultural uses of a sink and a tub, they should reject a bed as an alternative.

\section{EXPERIMENT 2}

\section{Method}

Participants. Eight 14-month-old infants were tested, 4 boys and 4 girls. They were recruited from the same source as in Experiment 1 . Their mean age was 14 months, 16 days (range $=14$ months, 3 days -14 months, 28 days).

Procedure. The same four properties along with the same modeling exemplars, props, and vocalizations used in Experiment 1 were tested again using different contrast sets. All the appropriate generalization objects were the same as those used in Experiment 1; only the inappropriate generalization objects were changed. Washing a dinner plate was tested with the sink and a bed. Sitting at a dinner table was tested with the chair and a small sedan with a flat top about the same height as the chair seat. Brushing hair was tested with the hairbrush and a spoon. Hammering pipes together was tested with the hammer and a cup. In each case it was physically as easy for infants to use the inappropriate as the appropriate object.

Infants were given an opportunity to explore the test items and prop before each property was tested. After each event was modeled by the experimenter, the modeling exemplar was removed from the table. The experimenter then brought out the test objects and placed them on either side of the infant with placement of appropriate and inappropriate exemplars counterbalanced across tasks. The prop (e.g., plate) was then placed in the infant's hand and the vocalization (e.g., "Wash the dishes") was repeated. The order in which the properties were administered was counterbalanced across participants. Scoring followed the same criteria as used in Experiment 1 . Coder reliability was $100 \%$.

\section{Results}

The mean number of first choices of appropriate and inappropriate objects during both baseline and generalization, along with the standard errors and percentage scores, are shown in the center portion of Table 1. As in Experiment 1, difference 
scores between the number of first choices to appropriate and inappropriate objects were calculated for each participant for both baseline and generalization; the means for these scores are shown in the center portion of Table 2 .

Generalization analyses. A one-way ANOVA was conducted on the difference scores with gender as the between-subjects variable. No significant effects were found. Next we used a $t$ test to see if the scores differed from zero. In contrast to the findings in Experiment 1, the 14-month-olds in this experiment were significantly more likely to generalize the modeled actions to appropriate than inappropriate objects. The mean difference score was 2.13 and was significantly greater than zero, $t(7)=7.20, p<.001$. A comparison of these data was then made with those of the 14-month-olds in Experiment 1. A $t$ test showed that the difference scores for Experiment $2(M=2.13)$ were significantly higher than those for Experiment $1(M=$ $-0.38), t(14)=3.76, p<.01$.

Baseline, comparison with generalization, and task analyses. The means in Table 1 suggest that infants showed a spontaneous preference for using the appropriate objects to carry out target actions during baseline. However, a $t$ test conducted on the difference scores for the baseline data showed that they did not differ significantly from zero, $t(7)=1.27, p=.24$. Because of the low power of the test, the conclusion of no preference for appropriate objects to use when carrying out these actions is tentative. As for the effects on choice behavior of modeling the correct response, although appropriate choices increased, the difference was only marginally significant, presumably because of the high amount of correct responding in baseline, $t(7)=2.05, p=.08$.

Finally, as in Experiment 1, we analyzed the data to see if some activities were demonstrated correctly more often than others. A one-way ANOVA with target action as a within-subjects measure was not significant $(p=.92)$, indicating that performance was roughly the same for each of the individual tasks.

\section{Discussion}

Even though the same events were modeled and tested for generalization in Experiments 1 and 2, the results were quite different. In Experiment 1, when related foils were used in the generalization test, 14-month-olds performed poorly, not seeming to recognize the difference in appropriateness of, say, a sink and a bathtub for washing dishes. However, in Experiment 2, in which the foils were less related, such as a bed versus a sink, performance was greatly improved. Even though the bed (with a headboard and footboard and slightly raised sides) formed a physical container, it was rarely used to imitate washing. Similarly, although the spoon had the same 
overall size and shape as the hairbrush and could be put to the hair as easily, the infants rarely used it to imitate brushing hair. It seems clear that at this age infants are not depending merely on simple physical descriptions (e.g., "largish container" or "hand-sized elongated object") or the affordances implied by these physical descriptions to determine the function of household objects. They may confuse a sink and a tub as appropriate for washing dishes, perhaps because they have seen both get filled with water or because washing of some sort takes place in both, but they do not confuse a sink and a bed despite their overall container-like shapes. ${ }^{2}$ Similarly, even though they are not clear about the different uses of various brushes, they know that spoons are not used for grooming hair, despite their rough similarity in shape. Thus, by 14 months infants have developed some fairly broad but not yet detailed conceptualizations of the functions of various household artifacts.

Because the inappropriate objects in this experiment came from very different categories than the appropriate objects, they were also perceptually more dissimilar to the objects used in modeling than was the case in Experiment 1. This may have made it easier for the infants to reject them as objects to be used in the generalization task. A purely perceptual explanation cannot tell the whole story, however. In Experiment 1, although the perceptual differences between appropriate and inappropriate objects were not as great, the inappropriate choices were also less similar in appearance to the modeled objects than the appropriate ones. Nevertheless, the infants chose them more often for their imitations, which is the opposite result from Experiment 2. For example, in Experiment 1 the two sinks looked more like each other than the bathtub, yet the children frequently chose the tub. A more likely explanation is that 14-month-olds simply do not pay much attention to the details of the objects they interact with and therefore are not sensitive to the role these details play in the activities in which the objects take part. This is another way of expressing the conclusion that at 14 months infants are not yet paying attention to basic-level distinctions, which crucially depend on parts, such as whether a container has a handle or a tool has a flat surface suitable for hammering. We know that they can see these differences (e.g., Behl-Chadha, 1996; Eimas \& Quinn, 1994), but they do not appear to attend to them with respect to the target actions.

\section{EXPERIMENT 3}

Experiments 1 and 2, in conjunction with our prior study of basic-level generalization (Mandler \& McDonough, 1998b), suggest that overgeneralized conceptions of both household artifacts and animals may be the rule at 14 months of age. At the

\footnotetext{
${ }^{2} \mathrm{An}$ interesting asymmetry worth further exploration is that infants this age did use both beds and tubs as places to imitate sleeping (Mandler \& McDonough, 1998b).
} 
same time, in several experiments we have found that infants seem to know more about vehicles than they do about either animals or household artifacts. First, from 7 to 11 months, infants differentiate cars from motorcycles and airplanes on the object-examination test, although they fail to differentiate different kinds of furniture or different kinds of mammals (Mandler \& McDonough, 1993, 1998a). Second, even though 14-month-olds show they have generalized vehicle properties across the entire domain, as illustrated by their using keys on airplanes and forklifts (Mandler \& McDonough, 1996), when given the choice they are more likely to choose another member of the same basic-level vehicle category for their imitations than a different basic-level vehicle category (Mandler \& McDonough, 1998b). The same selectivity is not shown for animals, suggesting again that infants are differentiating the vehicle domain earlier than some others.

Experiment 3 examines whether this seemingly more advanced understanding of different kinds of vehicles includes understanding that some vehicle properties are restricted to particular basic-level categories. We were able to find two basic-level vehicle properties that we expected 1-year-old infants to know: wearing helmets when riding a motorcycle (but not when riding in a car) and putting gas into a car (but not into a child's wagon). Although not strictly basic-level properties, because helmets are also used with bicycles and gasoline is used with several kinds of vehicles, they fulfill the spirit of basic-level properties in that they are more closely associated with some kinds than with others. (This looser association of basic-level properties with their respective basic-level categories seems to be fairly widespread. With the obvious exception of biological properties, many behaviors and actions associated with a given basic-level kind, such as a dog rather than a cat being on a leash, or drinking from cups rather than from pans, are occasionally extended to other kinds. In the cases here, however, infants would only have seen gasoline being put into cars, although just possibly into motorcycles, too. As for helmets, it is California state law that children wear helmets when riding bicycles or tricycles.) We also included two basic-level animal properties not only to ensure the replicability of our prior finding (Mandler \& McDonough, 1998b) that even at 19 months children are overgeneralizing basic-level animal properties, but also to assess the age at which this kind of mistake disappears.

\section{Method}

Participants. Twenty-four participants, 8 in each of three age groups, were tested: 14-, 19-, and 24-month-olds. They were recruited in the same fashion as in Experiments 1 and 2. The mean ages of each group were: 14 months, 13 days (range $=14$ months, 0 days -14 months, 23 days); 19 months, 10 days (range $=19$ months, 2 days -20 months, 0 days); and 24 months, 20 days (range $=24$ months, 2 days -24 months, 28 days). An equal number of boys and girls were tested at 19 and 24 
months of age; 5 boys and 3 girls were tested at 14 months of age. One additional participant was tested but not included in the final analysis due to unwillingness to imitate any of the actions either for warm-up or test.

Objects and properties tested. Participants were tested on four basic-level properties: two drawn from the animal domain and two from the vehicle domain. As in the previous experiments, the events were modeled using small replicas of real objects. The animal properties were eating a carrot and sitting on a nest. They were demonstrated by placing a carrot to the face of a white, domestic rabbit and placing a sparrow-like bird with outstretched wings in a small nest. The vehicle properties were wearing a helmet and putting in gasoline. They were demonstrated by the experimenter putting a helmet on a small doll in a seated position and placing the doll on an all-terrain vehicle (a three-wheeled motorcycle) and by placing a gasoline pump to the side of a sedan. (Because of the possible difficulty 14-month-olds might have with putting the helmet on the doll, for the baseline and generalization tests with this item the experimenter attached the helmet to the doll before handing it to the participants). The actions were accompanied by appropriate vocalizations that referred to the property tested but not the appropriate exemplar: "Yum, yum!" for eating a carrot; "In the nest!" for sitting on a nest; "Be safe!" for wearing a helmet; and "Put in gas!" The test exemplars were different from the modeling exemplars in terms of color, shape, and subordinate category. The test exemplars for the animal properties were a different rabbit and a hummingbird. The test exemplars for the vehicle property "wears a helmet" were a different motorcycle and car; the test exemplars for "puts in gas" were a different car and a child's wagon. (Although we would have liked to use the same basic-level kinds both as target and distractor as we did for the animals, it did not work for this particular vehicle property; the motorcycle could not be used as a distractor to test putting in gas because it also uses gasoline.)

Procedure. The same procedure followed in Experiments 1 and 2 was used. The two warm-up tasks were putting a plastic star into a container, covering and shaking it, and stirring a spatula in a frying pan. Participants were encouraged to imitate these tasks and were praised for doing so. Following the warm-up, the four properties were assessed in counterbalanced sequence. For each property, first baseline was assessed, and then the test exemplars were put away, leaving the prop on the table. The experimenter brought out the modeling object and modeled the property three times with accompanying vocalizations. The modeling object was then removed from the table. The test exemplars were brought out and placed on each side of the infant. The prop was handed to the infant with the accompanying vocalization (e.g., "Be safe"). Again, scoring took place from the videotapes and reliability between the two coders who coded every participant exceeded $98 \%$. 


\section{Results}

The mean number of first choices of appropriate versus inappropriate objects to carry out target actions in baseline and generalization for the three age groups are shown in the bottom portion of Table 1, along with standard errors and percentage scores. The mean difference scores, calculated by subtracting the number of inappropriate first choices from appropriate first choices for each participant in each condition, are shown in the bottom portion of Table 2.

Generalization analyses. An ANOVA for repeated measures was conducted on the difference scores with age (14, 19, and 24 months) and gender as between-subjects factors and domain (animal, vehicle) as a within-subjects factor. No effects of gender were found. There was a main effect for age, $F(2,18)=5.48, p<$ .05 , which was qualified by an interaction with domain, $F(2,18)=2.72, p=.05$. Follow-up analyses were first conducted on the data from the animal domain and showed a significant effect for age, $F(2,21)=4.85, p<.05$. Independent samples $t$ tests showed that the 24-month-olds had significantly larger difference scores $(M=$ 1.50) than the 19-month olds $(M=0.00)$, but the 19-month-olds and the 14 -month-olds $(M=0.75)$ did not significantly differ, $t(14)=1.42, p=.18$. Analyses conducted on the data from the vehicle domain also showed an effect for age, $F(2,21)=4.26, p<.05$. Follow-up analyses showed no significant differences between the 24- and 19-month-olds ( $M=1.63$ and 1.38, respectively); however, both the older ages showed larger difference scores than the 14-month-olds $(M=0.50)$, $t(14)=3.00, p=.01$, for the comparison between 24 and 14 months and, $t(14)=$ $2.08, p=.06$, for the comparison between 19 and 14 months.

To assess the import of these difference scores, we compared them to the chance expectation of zero for choosing appropriate over inappropriate exemplars. The results showed that the difference scores of the 14-month-olds were not significantly greater than zero for either the animal or vehicle domain properties (both $p$ s $>$.08). For the 19-month-olds, generalization of animal properties was still at chance, but generalization of vehicle properties to the appropriate exemplar was now significantly greater than chance, $t(7)=4.25, p<.01$. Finally, the 24-month-olds generalized both animal and vehicle properties to the appropriate exemplar significantly more often than chance, $t(7)=5.61, p<.01$, for animals and, $t(7)=6.18, p<.001$, for vehicles. Thus, as indicated in Table 1 , by 19 months of age, children were generally correct on the vehicle properties but not until 24 months of age were they generally correct on the animal properties.

Baseline, comparison of baseline to generalization, and task analyses. As can also be seen in Table 1, few preferences were found for using the appropriate exemplars when carrying out target activities at baseline. The difference scores were evaluated against the chance expectation of zero by 
means of $t$ tests. No significant effects were found for either domain at any age. In addition, an ANOVA on the differences scores with age and gender as between-subjects factors and domain as a within-subjects factor, showed no significant effects.

To examine the effect of modeling on choice of appropriate objects for the actions, an ANOVA was conducted on number of appropriate first choices (range: $0-2)$, with age (14, 19, and 24 months) and gender as between-subjects variables and domain (animal, vehicle) and condition (baseline, generalization) as within-subjects variables. There was a main effect for condition, qualified by a marginal interaction with age, $F(2,18)=3.38, p<.06$. Follow-up analyses were conducted on each age group, showing that appropriate demonstrations increased significantly from baseline to generalization for all three ages (all $p \mathrm{~s}<.05$ ). Further one-way ANOVAs on baseline and generalization showed no age effects in baseline, $F(2,21)=1.46, p=.26$. However, there was considerable improvement in generalization performance with age, $F(2,21)=9.51, p<.001$. Tukey tests at $p$ $=.05$ showed significantly better performance for the 19- and 24-month-olds than for the 14-month-olds. There was also a three-way interaction between condition, domain, and gender, $F(1,18)=5.41, p<.001$. To analyze this interaction we broke down the data by domain, analyzing the animal and vehicle properties separately. For the animal properties a Condition $\times$ Gender interaction was found, $F(1,22)=$ $4.55, p<.05$. The girls produced significantly more of the target actions at generalization $(M=1.45)$ than at baseline $(M=0.36)$, but the boys did not $(M=1.0$ for generalization and $M=0.69$ for baseline). This effect resulted from the boys producing somewhat more animal properties in baseline and somewhat fewer in generalization. For the vehicle properties, there was a main effect for condition, $F(1$, 22 ) $=76.66, p<.001$, but no effect for gender. A mean of 1.34 properties (of 2 ) were demonstrated in generalization, whereas the mean for baseline was only 0.14 . Thus, there was a high percentage of appropriate generalizations for the vehicle properties but relatively few during baseline, suggesting that the children were familiar with the actions, but they were not prepotent in their spontaneous play.

Finally, we examined the four tasks to see if some activities were more likely to be performed correctly than others. We entered the number of correct first choices (combining baseline and generalization, giving a range of scores from 0-2) into an ANOVA with target action as a within-subjects factor and age as a between-subjects factor. A main effect was found for target action, $F(3,19)=12.22, p<.001$. Follow-up $t$ tests showed that within the animal domain, putting a bird in the nest $(M=1.17)$ was more apt to be correctly performed than feeding a rabbit a carrot $(M$ $=0.58), t(23)=3.98, p<.001$. Similarly, in the vehicle domain, placing the rider with a helmet onto a motorcycle $(M=1.00)$ was correctly performed more often than putting gas in a car $(M=0.50), t(23)=4.15, p<.001$. There were no significant differences between the two domains; in each domain, one property was more likely to be performed correctly than the other. 


\section{Discussion}

This experiment investigated the ages at which children learn some common basic-level characteristics of animals and vehicles. Once again, we found more advanced behavior in the vehicle domain than in the animal domain, in that by 19 months the participants were much more likely to generalize correctly basic-level characteristics of vehicles than basic-level characteristics of animals. This finding indicates that the good performance by 19-month-olds on vehicles and on the household artifacts studied in Experiment 1 is not merely due to an increase in a general ability to perceptually match the stimuli used in modeling. If the improvement in age were due only to increasing ability to perceptually match choice of stimuli to those used for modeling, then the participants should have performed as well with animals as with vehicles, but they did not. If anything, the perceptual differences among the animals were greater than the differences among the vehicles.

This rejection of perceptual matching ability as an explanation for the data in these experiments is not meant to preclude the hypothesis that improved accuracy of generalization with age is due in part to increased attention to the perceptual details of various animals and artifacts. The data from Experiment 3 merely suggest that less attention is paid to the details of what animals look like than what vehicles look like and that this relative indifference to detail persists longer. At present we have no information about the frequency with which children experience the characteristic properties of animals and vehicles, or even whether they are comparable in what they demand in the way of understanding. However, the data add to the frequent if not always significant finding in our prior experiments of earlier conceptual differentiation of vehicles into separate kinds than that with animals (Mandler \& McDonough, 1993; Mandler \& McDonough, 1998b). It is possible that the social, interactive nature of animals is attention-demanding enough to lessen attentiveness to details of their physical appearance.

\section{GENERAL DISCUSSION}

This series of experiments, in conjunction with similar results reported previously (Mandler \& McDonough, 1998b), shows that infants do not at first appreciate the differences among many basic-level concepts within the domains of animals, household artifacts, and vehicles. On the contrary, these data, in conjunction with our previous work on this topic, indicate that the earliest concepts about these objects are at a rather global level and that learning the details that distinguish one basic-level concept from another is a later achievement. The rate at which knowledge accumulates about basic-level characteristics varies somewhat from domain to domain and to some extent with gender. In particular, within-domain knowledge seems to accumulate more slowly for animals than for artifacts (at least in the Cali- 
fornia culture studied). After watching appropriate animal events such as feeding a carrot to a rabbit, both 14- and 19-month-olds often fed the carrot to a bird instead of to another rabbit, and after watching a bird put in a nest they often put a rabbit in the nest instead of another bird. Not until 24 months did they reliably demonstrate the appropriate behaviors for these different animals. In the domain of household artifacts, on the other hand, although 14-month-olds did not differentiate sinks from bathtubs, hairbrushes from toothbrushes, hammers from wrenches, or chairs from toilets, by 19 months most participants performed correctly on these common household objects. Both of these findings are consistent with those reported in Mandler and McDonough (1998b), in which earlier differentiation was found for household artifacts than for animals and plants. In the prior work, both 14- and 19-month-olds generalized chewing on bones from dogs to geese, and smelling flowers to trees. Fourteen-month-olds were also poor at differentiating household artifacts; they imitated drinking from a frying pan as often as from a cup and put a doll to sleep in a tub as often as in a bed. However, by 19 months they were more likely to restrict their behaviors in the household domain to the appropriate basic-level classes.

The data reported here also suggest that boys lag somewhat behind girls in developing knowledge in the household domain. However, this finding did not carry over to vehicles, as shown in Experiment 3.

There, boys performed as accurately on vehicles as girls at 19 months. Experiment 3 was also useful in showing that the improvement in performance that occurred with age is unlikely to be due solely to an increase in the ability to match the objects the modeler uses because the same participants behaved differently toward animals and vehicles. Poor performance was general at 14 months, but at 19 months performance on vehicle properties was generally accurate, whereas performance on animal properties was still poor.

The third experiment also indicated that knowledge about subclasses of vehicles develops at approximately the same rate as for household artifacts, in that 19-month-olds were largely correct for both domains. However, we note that in one respect behavior toward vehicles was more advanced; the least amount of inappropriate behavior in any of these experiments occurred for vehicles, even among 14-month-olds, and for boys as well as girls. Direct comparisons with a wider range of properties would be needed to test this hypothesis more thoroughly.

It is of some interest that success in showing differentiated behavior to vehicle basic-level categories depends on the task. In object examining, which is the easiest of the three categorizing tasks we have used, infants as young as 7 months differentiated cars from motorcycles (Mandler \& McDonough, 1993). In sequential touching, which is a more difficult task, cars were not differentiated from motorcycles until 24 months and trucks even later (Mandler, Bauer, \& McDonough, 1991). In generalized imitation, sensitivity to differences among cars, trucks, and motorcycles was shown by 14-month-olds when domainwide characteristics such as be- 
ing keyed and giving rides were modeled, but the work reported here suggests that not until 19 months are children adept at associating specific basic-level vehicle properties appropriately. This is a somewhat mixed picture compared to the consistent failure of children up to 24 months of age or even older to differentiate mammals on all the tasks we have used. It is also a more mixed picture compared to the failure of infants to differentiate types of furniture on the object examination and sequential touching tests (Mandler et al., 1991; Mandler \& McDonough, 1998a). Overall, however, the data suggest that vehicles may be differentiated into subtypes somewhat sooner than furniture. That is, among artifacts, it may be that information about vehicles is not learned unusually rapidly but that information about furniture is learned relatively slowly. The database on these issues is still not large, and further work will be required to decide among these alternative interpretations. In any case, the data collected to date indicate that artifacts of all types are differentiated sooner by California children than are animals (and plants).

The frequent occurrence of overgeneralized behaviors at 14 months should not be taken to mean that infants of this age know nothing about the characteristic properties of the household artifacts that were studied. Experiment 2 showed that they were successful in restricting their generalizations when the foils were less closely related to the correct choices. Thus, although the infants used a toothbrush to groom hair, they did not use a spoon for this purpose, and although they washed dishes in a bathtub, they did not wash dishes in a bed. These findings support our previous results showing that the concepts of infants of this age are rather broad and relatively undifferentiated (e.g., Mandler \& McDonough, 1996, 1998b). The results, however, provide more precise information about these conceptualizations. For example, although 14-month-olds do not distinguish between beds and tubs as places to sleep (Mandler \& McDonough, 1998b), they do distinguish between beds and sinks as places to wash dishes. This kind of finding suggests that differentiation of the functions of various household objects may be learned in a piecemeal fashion, so that anomalous associations can exist along with correct associations. Acquiring true basic-level understanding appears to be a gradual process. Children might first learn that certain kinds of objects are containers (Baillargeon, Kotovsky, \& Needham, 1995); then that small containers, whether cups or frying pans, are used for drinking and large containers are used for washing and sleeping; then that some large containers are used for sleeping and others only for washing; and, finally, the specifics that differentiate tubs from sinks. This specific developmental progression is speculative, of course, because we have only collected some of the relevant data. More generally, familiarity with the various subclasses of the global domains we have studied may affect the rate at which they become differentiated. Overall, however, the general developmental trend of conceptualization advancing downward from the global to the basic level has been found consistently in our research on this topic (e.g., Mandler et al., 1991; Mandler \& McDonough, 1998a, 1998b). 
Language acquisition may also affect the course of conceptual differentiation during this period (Gopnik \& Meltzoff, 1992). However, the causal relation between language learning and conceptual differentiation is far from clear. The second year is a time of considerable overextension in basic-level terms, not only in production (Clark, 1983) but also in comprehension (McDonough, 1997). Overextension in comprehension is predictable from overly global concepts. It is less clear, however, whether the narrowing down of object terms to their approximate adult extension is due to prior conceptual differentiation or whether adults' consistent use of different labels for certain objects encourages the attention to detail that is required for basic-level conceptual learning. In either case, a large part of the improvement found in these experiments between 14 and 24 months seems to be that more attention is being paid to the perceptual details that define basic-level classes. This finding is consistent with the gradual increase in reliance on shape when learning names for new objects (Jones \& Smith, 1993). In addition, the older children's better comprehension of the language that accompanied the modeling may have increased the amount of imitation that was carried out (Bates, Thal, Fenson, Whitesell, \& Oakes, 1989).

It is worth noting one implication of the fact that the older children did more imitation in general (in addition to making fewer incorrect choices) than the younger ones. It is possible that the imitation technique may tend to underestimate somewhat the knowledge of the 14-month-olds, because they must imitate if they are to demonstrate that knowledge. At the same time, within the 14-month age group, we have found lesser rates of imitation when testing basic-level properties (Experiments 1 and 3) than when testing global properties (Mandler \& McDonough, 1996, Experiments 1 and 2). It appears, therefore, that some of the lower rate of imitation among the 14-month-olds for basic-level activities is due to their relative lack of knowledge about the properties in question. In the face of uncertainty, some infants may choose not to imitate at all.

Finally, the current data highlight a point we have made previously (Mandler \& McDonough, 1998b): Associative learning is dependent on the interpretation of the meaning of objects-not on their physical features. Infants and young children have observed birds in nests (or have been shown pictures of birds in nests) but, nevertheless, associate nests with other animals as well. They have seen people drink from cups and mugs but, nevertheless, associate drinking with other containers such as pans. They have been washed in tubs and observed washing dishes in sinks but associate washing dishes in tubs as well. These and other examples, such as associating sleeping with fish, keying with airplanes (Mandler \& McDonough, 1996), or chewing bones with birds, strongly suggest that associative learning is not under the control of object features but object meaning. Thus, if a dog is interpreted as an animal, then observed behaviors of dogs such as sleeping or chewing bones become associated not just with dogs but with animals and, hence, are generalized to fish and birds. This kind of finding has ramifications beyond under- 
standing the nature of early concepts; it suggests that the common psychological view of associations as being controlled by physical similarity is in need of modification. More attention needs to be paid to the role played by conceptual similarity in forming the associations that build our knowledge base.

\section{ACKNOWLEDGMENTS}

Preparation of this article was supported in part by National Science Foundation Research Grant SBR-970895 and PSC-CUNY Research Grant 69685-00-29. We thank Lisa Hoag for help in testing and data analysis.

\section{REFERENCES}

Baillargeon, R., Kotovsky, L., \& Needham, A. (1995). The acquisition of physical knowledge in infancy. In G. Lewis, D. Premack, \& D. Sperber (Eds.), Causal understandings in cognition and culture (pp. 7-116). Oxford, England: Oxford University Press.

Bates, E., Thal, D., Fenson, L., Whitesell, K., \& Oakes, L. (1989). Integrating language and gesture in infancy. Developmental Psychology, 25, 1004-1019.

Bauer, P. J., \& Dow, G. A. (1994). Episodic memory in 16- and 20-month-old children: Specifics are generalized but not forgotten. Developmental Psychology, 30, 403-417.

Bauer, P. J., \& Thal, D. (1990). Scripts or scraps: Reconsidering the development of sequential understanding. Journal of Experimental Child Psychology, 50, 287-304.

Behl-Chadha, G. (1996). Superordinate-like categorical representations in early infancy. Cognition, 60, 104-141.

Clark, E. V. (1983). Meanings and concepts. In P. H. Mussen (Series Ed.) \& J. H. Flavell \& E. M. Markman (Vol. Eds.), Handbook of child psychology: Vol. 3. Cognitive development (pp. 787-840). New York: Wiley.

Eimas, P. D., \& Quinn, P. C. (1994). Studies on the formation of perceptually based basic-level categories in young infants. Child Development, 65, 903-917.

Gopnik, A., \& Meltzoff, A. N. (1992). Categorization and naming: Basic-level sorting in eighteen-month-olds and its relation to language. Child Development, 63, 1091-1103.

Jones, S. S., \& Smith, L. B. (1993). The place of perception in children's concepts. Cognitive Development, 8, 113-139.

Killen, M., \& Uzgiris, I. C. (1981). Imitation of actions with objects: The role of social meaning. Journal of Genetic Psychology, 138, 219-229.

Lassaline, M. E., Wisniewski, E. J., \& Medin, D. L. (1992). Basic levels in artificial and natural categories: Are all basic levels created equal? In B. Burns (Ed.), Percepts, concepts and categories (pp. 327-378). Amsterdam: Elsevier.

Legerstee, M. (1992). A review of the animate-inanimate distinction in infancy: Implications for models of social and cognitive knowing. Early Development and Parenting, 1, 59-67.

Mandler, J. M. (1992). How to build a baby: II. Conceptual primitives. Psychological Review, 99, 587-604.

Mandler, J. M. (1997). Development of categorisation: Perceptual and conceptual categories. In G. Bremner, A. Slater, \& G. Butterworth (Eds.), Infant development: Recent advances (pp. 163-189). Hove, England: Lawrence Erlbaum Associates, Inc. 
Mandler, J. M., Bauer, P. J., \& McDonough, L. (1991). Separating the sheep from the goats: Differentiating global categories. Cognitive Psychology, 23, 263-298.

Mandler, J. M., \& McDonough, L. (1993). Concept formation in infancy. Cognitive Development, 8, 291-318.

Mandler, J. M., \& McDonough, L. (1996). Drinking and driving don't mix: Inductive generalization in infancy. Cognition, 59, 307-335.

Mandler, J. M., \& McDonough, L. (1998a). On developing a knowledge base in infancy. Developmental Psychology, 34, 1274-1288.

Mandler, J. M., \& McDonough, L. (1998b). Studies in inductive inference in infancy. Cognitive Psychology, 37, 60-96.

McDonough, L. (1997, April). Overextension in the production and comprehension of basic-level nouns in two-year-olds. Poster presented at the meeting of the Society for Research in Child Development, Washington, DC.

McDonough, L., \& Mandler, J. M. (1998). Inductive generalization in 9- and 11-month-olds. Developmental Science, 1, 227-232.

Mervis, C. B., \& Rosch, E. (1981). Categorization of natural objects. Annual Review of Psychology, 32, $89-115$.

Molina, M., Spelke, E. S., \& King, D. (1996, April). The animate-inanimate distinction in infancy: Sensitivity to distinctions between social interactions and object manipulations. Poster presented at the Tenth International Conference on Infant Studies, Providence, RI.

Nelson, K. (1974). Concept, word, and sentence: Interrelations in acquisition and development. Psychological Review, 81, 577-584.

Piaget, J. (1951). Play, dreams, and imitation in childhood. New York: Norton.

Poulin-Dubois, D., Lepage, A., \& Ferland, D. (1996). Infants' concept of animacy. Cognitive Development, 11, 19-36.

Quinn, P. C., Eimas, P. D., \& Rosenkrantz, S. L. (1993). Evidence for representations of perceptually similar natural categories by 3-month-old and 4-month-old infants. Perception, 22, 463-475.

Rosch, E., Mervis, C. B., Gray, W., Johnson, D., \& Boyes-Braem, P. (1976). Basic objects in natural categories. Cognitive Psychology, 3, 382-439.

Tanaka, J. W., \& Taylor, M. (1991). Object categories and expertise: Is the basic level in the eye of the beholder? Cognitive Psychology, 23, 457-482. 